\title{
CURRENT STATE OF IN-CABINET RESPONSE SPECTRA FOR SEISMIC QUALIFICATION OF EQUIPMENT IN NUCLEAR POWER PLANTS
}

\author{
Abhinav Gupta ${ }^{1}$, Sung-Gook Cho ${ }^{2}$, Kee-Jeung Hong ${ }^{3}$, Minsoo Han ${ }^{4}$ \\ ${ }^{1}$ Professor, Dept. of Civil, Construction and Environmental Engineering, NC State University, USA \\ ${ }^{2}$ Director, R\&D Center, Innose Tech Co. Ltd., Korea \\ ${ }^{3}$ Professor, School of Civil and Environmental Engineering, Kookmin University, Korea \\ ${ }^{4}$ Researcher, R\&D Center, Innose Tech Co. Ltd., Korea
}

\section{INTRODUCTION}

South Korea experienced its biggest earthquake in recent history on September 12, 2016. The Gyeongju earthquake of magnitude $\left(\mathrm{M}_{\mathrm{w}}\right) 5.4$ has initiated a significant activity in the area of seismic safety for nuclear power plants. Initial reports indicate that the nature of this earthquake is similar to the recent findings from geophysical investigations in Central and Eastern United States (CEUS), i.e., the earthquake ground motions contain not only the low frequency content but also some high frequency content. As is the case with most of the nuclear plants in CEUS, the safe shutdown earthquake (SSE) for most of the Korean plants have only low frequency content (below $10 \mathrm{~Hz}$ ). Therefore, the nuclear plants in Korea are beginning to conduct a detailed seismic safety assessment of their structures, systems, and components (SSC). In the context of seismic safety assessment, high frequency motions are not likely to cause damage to structural systems as the displacements associated with such motions are relatively very small. Yet, past experience around the world has shown that electrical instruments such as relays, breakers, and contact switches can exhibit loss of functionality due to high frequency motions. These instruments must continue to operate as intended during and after an earthquake. The earthquake motion exhibited by such instruments is highly dependent on the dynamic characteristics of the cabinets and control panels on which they are mounted. The earthquake motion used in shake table testing of such instruments is called in-cabinet response spectra (ICRS). In this paper, we summarize the characteristics of Gyeongju earthquake and review the various methods used for evaluating ICRS including some of the recent simple methods that have been validated against test data. The paper focuses on evaluating applicability of each method and its appropriateness for practical implementation. Technical gaps are also identified in order to assist with future research.

\section{HIGH FREQUENCY CONTENT IN GYEONGJU EARTHQUAKE}

Historically, the past earthquakes near Gyeongju occurred primarily on the eastern side of the Yangsan fault as shown in Figure 1a. On the contrary, the main shock of the Gyeongju earthquake occurred slightly west of the Yangsan fault (Sun et al., 2016). Since there are Wolsong, Kori and Hanwool nuclear power plants near the epicentre of the Gyeongju earthquake, it is important to develop an understanding of the resiliency of these nuclear plants against such type of earthquakes. Among these nuclear power plants, the Wolsong nuclear power plant is located nearest to the epicentre, only $27 \mathrm{~km}$ away. The Gyeongju earthquake resulted from a strike-slip fault in the Yangsan fault zone, and the depth of the hypocentre is estimated to be $13 \mathrm{~km}$ (Sun et al., 2016). This hypocentre of the Gyeongju earthquake is relatively deeper than the average hypocentre depth, approximately $10 \mathrm{~km}$, for other strong historical Korean earthquakes with a magnitude of more than 5.0 (Kim and Park, 2016). The Gyeongju earthquake resulted in 23 injuries and 9,368 cases of damages as of October 4, 2016, which caused approximately 10 million US dollars of property damage (KMPSS, 2016). Property damage of about 8.45 out of 10 million US dollars occurred in the geographical 
region of Gyeongju. In order to examine the effects of the characteristics of Gyeongju earthquake particularly on the electrical equipment and infrastructure, three acceleration time histories recorded at the Myeonggye-ri station (MKL), Ulsan station (USN), and Deokjeong-ri station (DKJ) are considered in this paper. These recording stations are close to the epicentre of the Gyeongju earthquake as shown in Figure $1 \mathrm{~b}$. The response spectrum at each of these sites for three different directions are compared with the RG 1.60 design spectrum of $0.3 \mathrm{~g}$ PGA. The RG 1.60 spectrum is representative of a safe shutdown earthquake in many nuclear power plants (USNRC, 2014). Furthermore, the Korean standard design response spectrum (KMLIT, 2017) of $0.154 \mathrm{~g}$ PGA in horizontal direction and $0.103 \mathrm{~g}$ PGA in vertical direction are also compared with these curves as shown in Figure 2. Table 1 gives the peak ground acceleration (PGA) in each direction as well as the distance from epicentre for the three stations. As shown in Figure 2, the Gyeongju earthquake records contains a significant amount of high frequency motion, i.e. greater than 6.7 $\mathrm{Hz}$ (natural time period less than $0.15 \mathrm{sec}$ ). As stated earlier, the high-frequency content in the ground motion has the potential to interrupt the continued functionality of electrical components such as relays which in turn govern the reliable performance of equipment in major infrastructure. Therefore, relevant measures are needed to evaluate the reliability and safety of such systems during future events. This is especially so in case of nuclear plants because the loss of functionality for a simple electrical device like a relay can lead to major societal and economic disasters.

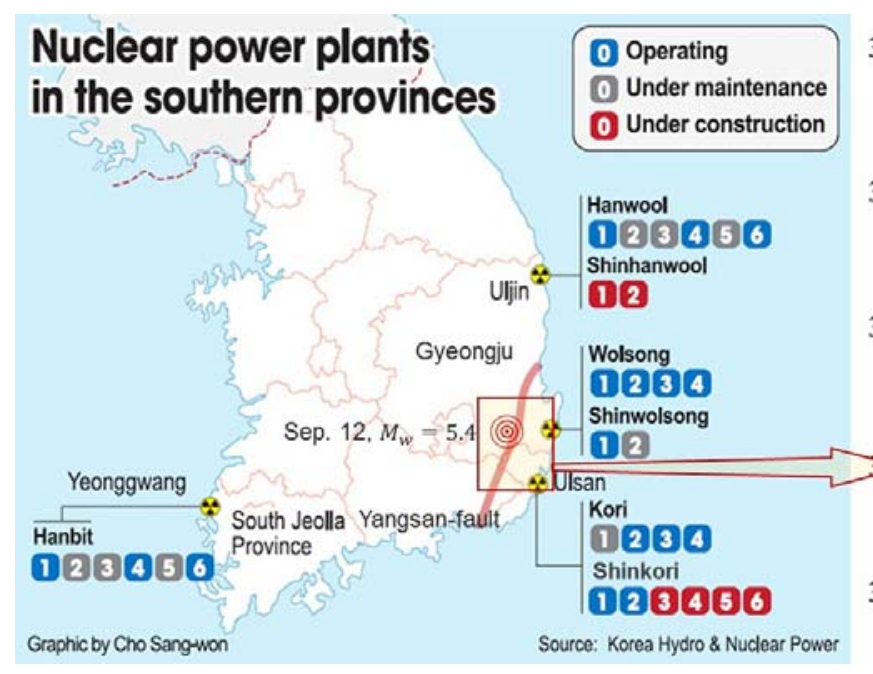

(a) The Gyeongju earthquake (red circles), the Yangsan fault (red solid line) and nuclear power plants nearby (nuclear symbols)

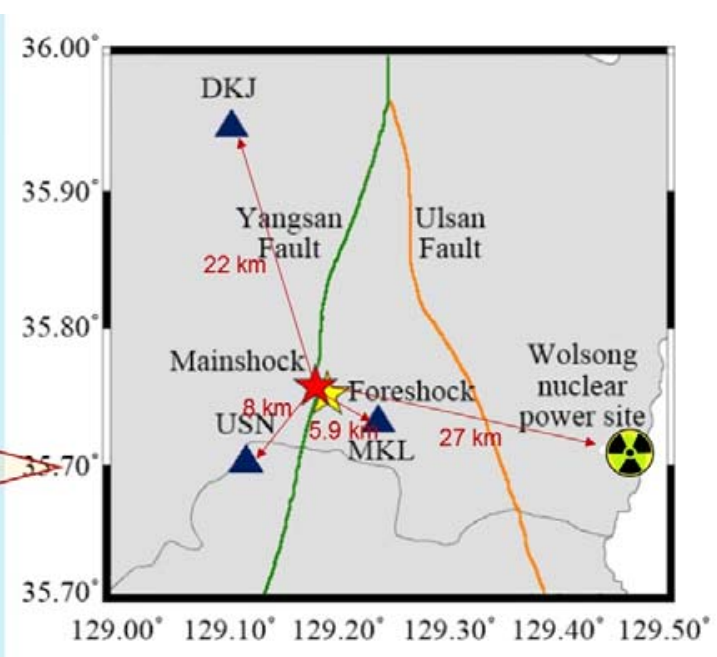

(b) The epicentre of the Gyeongju earthquake (red star) and the observation stations nearby (filled triangles)

Figure 1. The epicentre of the Gyeongju earthquake and the observation stations

Table 1: The locations and PGA of the observation stations

\begin{tabular}{|c|c|c|c|c|c|c|}
\hline \multirow{2}{*}{ Station } & \multirow{2}{*}{ Latitude } & \multirow{2}{*}{ Longitude } & \multirow{2}{*}{$\begin{array}{c}\text { Distance } \\
(\mathrm{km})\end{array}$} & \multicolumn{3}{|c|}{ PGA $(\mathrm{g})$} \\
\cline { 5 - 7 } & & & & $\mathrm{EW}$ & $\mathrm{NS}$ & VT \\
\hline MKL & 35.7322 & 129.2420 & 5.86 & 0.285 & 0.257 & 0.088 \\
\hline USN & 35.7024 & 129.1232 & 8.23 & 0.404 & 0.351 & 0.213 \\
\hline DKJ & 35.9468 & 129.1089 & 22.15 & 0.078 & 0.092 & 0.057 \\
\hline
\end{tabular}




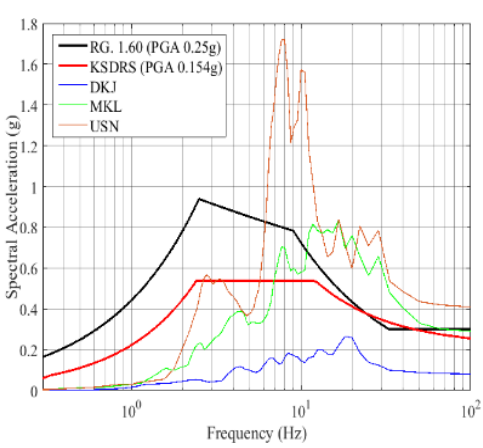

(a) EW direction

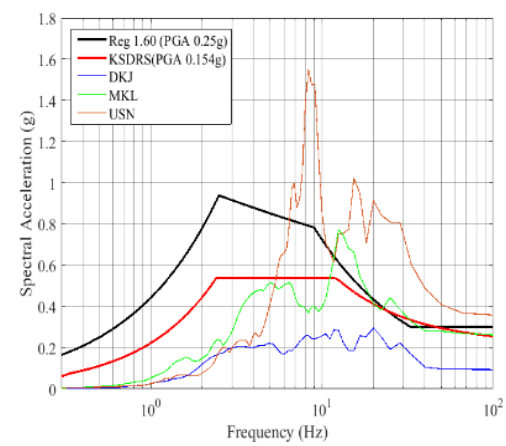

(b) NS direction

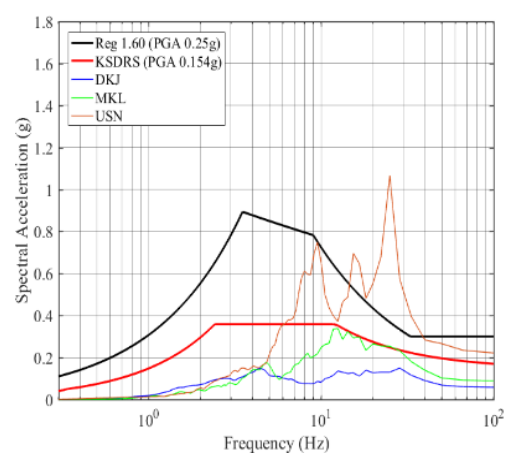

(c) Vertical (VT) direction

Figure 2. Comparison between the design $\&$ the recorded response spectra

\title{
CURRENT PRACTICE OF EVALUATING IN-CABINET RESPONSE SPECTRUM
}

\author{
EPRI NP-7146-SL: Background and Limitations
}

Two simple methods are proposed in EPRI NP-7146-SL guidelines (EPRI, 1995). These methods are based on in-situ modal testing of cabinets and control panels. However, these studies focus on evaluating maximum amplifications inside a cabinet and do not attempt to identify significant features of the cabinet dynamic behaviour. These methods can give unrealistic spectra that are excessively conservative in most cases. The methods are based on in-situ modal test data of 45 cabinets and can be used for only those cabinets that satisfy a specified set of caveats. In one of the two, the screening factor method, peak floor spectral acceleration is amplified by a factor of 4.5 to obtain the peak incabinet acceleration. The maximum value of resulting amplified acceleration is used as ICRS in the complete frequency range of interest. This factor was developed analytically using several incabinet spectra at the location of maximum amplification for three different input floor response spectra using cabinet dynamic properties evaluated from in-situ tests. They modified the narrow-band incabinet spectra to obtain broad-band clipped-peak spectra using knockdown factors of up to 0.6 (up to $40 \%$ clipping). For each of the three input floor response spectra, they calculated an 85-percentile incabinet spectrum curve and the corresponding amplification factor. The recommended value of 4.5 is close to the highest value of 4.8 among the three amplification factors. It has been found that even though knockdown factors are used to evaluate the screening factor of 4.5 , this method can give excessively conservative spectral accelerations for most situations. Conservatism is introduced by including amplification at locations where an instrument may never be mounted. The screening factor is not intended to be a precise number for evaluating incabinet spectra as such.

For cabinets in which the results of screening factor method are too conservative, a second method called the "simplified analysis" method is recommended. In this method, the fundamental cabinet frequencies, frequency dependent maximum pseudo participation factors, and the modal damping ratios from in-situ modal tests of 45 cabinets are used. Several incabinet spectra are generated analytically using these dynamic properties for a range of cabinet fundamental frequencies. The final spectrum is obtained by enveloping the generated spectra for the 45 tests. The maximum pseudo participation factors used in the analytical solution are not for the particular cabinet under consideration. Further, there is no good way of estimating the fundamental frequency of the cabinet. For most cases, unrelated high values of maximum pseudo participation factors and enveloping of several individual incabinet spectra introduces excessive conservatism.

Every utility understands the limitations and approximations in using the SQUG factors or EPRI NP-7146 SL guidelines. However, the premise is that the factors are in general quite conservative and if a relay worked well during shake table testing at acceleration levels (across a wide band of frequency - pretty 
much a flat wide ICRS) which is higher than the ICRS generated by using the factor then the relay is considered qualified.

EPRI NP-7146 SL is based on the in-situ testing of an initial set of 45 cabinets. The in-situ testing was used to generate modal properties which were then used to generate in-cabinet amplifications analytically. The amplifications generated in this study varied widely and some of the factors were as high as 20 which were clearly seen as unreasonable and it was identified that some of the factors pertain to locations that are not the location of any relay or other instruments. To reduce the high amplification obtained from the in-situ testing, EPRI NP-7146 SL methodology performs what is termed as "clipping" of spectra. This "clipping" is conducted in accordance with clipping factors to reduce the sharp narrow banded peaks of ICRS. The formulas for clipping factors were generated in 1980s based on theoretical work using SDOF primary -SDOF secondary system subjected to sinusoidal sine-beat type input motions.

It has been noted by many researchers that the set of cabinets used in the study that forms the basis for EPRI NP-7146 SL are only a very small representative of the type of cabinets found in most plants. It has also been acknowledged that the amplifications in other cabinets can be quite different from what forms the basis of EPRI 7146 SL. The work by SQUG was focused on overcoming this limitation by including more cabinet types and broadening the applicability of amplification factors. The SQUG effort focused on collecting a wider set of database from the various in-situ testing, shake table testing, and experience data on the performance of relays and electrical equipment. Subsequent research at NC State University identified that in some cases the ICRS generated by using the constant factors can be very conservative even if the smallest factors are used (Gupta et al., 1999). The same research also showed that in other cases the ICRS can be unconservative even if the largest factors are used.

In most cases, the fundamental mode of the cabinet is used to generate the amplifications. Subsequent research has shown that in many cabinets the fundamental mode is not necessarily the significant mode (Gupta et al., 1999). The most significant mode is typically the local mode of the panel or the frame on which a relay is mounted. It has also been shown that most analytical studies particularly those based on finite element analysis consider the cabinets to be fixed at their base. However, this is not true for cabinets that are anchored to the floor. Such anchored cabinets can have minor rocking/rattling of the whole cabinet and the behaviour at the mounting arrangement is quite complex and nonlinear (Yang et al., 2002). It is also noted that in many cases, the mounting arrangement used in the shake table testing of actual cabinets is not identical to the one used for in-situ anchorage. This difference can lead to a significant change in ICRS for some cases.

\section{Simplified Method: Ritz vector Approach}

Based on the discussion presented above, it can be observed that the significant cabinet mode evaluated from a finite element analysis and validated against experimental data represents a global cabinet rocking superimposed with the local mode of the plate or frame on which the instruments are mounted. A Ritz vector approach, in which the significant cabinet mode is taken as global rocking superimposed with local plate or frame modes, would give accurate results, i.e. the displacement $u$ at a given instrument location can be expressed as:

$$
u(\xi, \eta, t)=x_{r}(t) \phi_{r}(\eta)+x_{g}(t) \phi_{g}(\eta)+x_{l}(t) \phi_{l}(\xi, \eta)
$$

where $\phi_{r}(\eta)$, represents the Ritz vector for the rigid body rocking mode; $\phi_{g}(\eta)$ represents the Ritz vector for the global cantilever-type mode; and $\phi_{l}(\xi, \eta)$ represents the Ritz vector for the local mode shape of the plate or frame on which the instrument is mounted. Consistent with the coordinate system used in Gupta et al. (1999), the symbols $\xi$ and $\eta$ denote the horizontal and vertical coordinates whereas $x_{r}(t), x_{g}(t)$, and $x_{l}(t)$ represent the normal coordinates as a function of time $t$, for rocking, global bending, and local modes, respectively. A detailed discussion on the selection of Ritz vectors is presented in Gupta et al. (1999) and Gupta and Yang (2002). For a cabinet of height L, the Ritz vector for the rigid body rocking mode can be simply taken as 


$$
\phi_{\mathrm{r}}(\eta)=\frac{\eta}{L}
$$

Thus, equation 1 linearly transforms the motion at a given instrument location into an equivalent 3-DOF system. In situations where a global bending does not exist, the transformation results in an equivalent 2DOF system as the term corresponding to $\phi_{g}(h)$ drops out of equation 1 . The Ritz vector for the local mode shape $\phi_{l}(\xi, \eta)$ can be determined as explained later in this paper. Eigenvalue problem for this equivalent simple system can be written as

$$
\mathbf{K X}=\omega^{2} \mathbf{M X}
$$

where $\mathbf{K}$ and $\mathbf{M}$ are the equivalent stiffness and mass matrices, respectively, and $\mathbf{X}$ the vector of generalized coordinates such that

$$
\mathbf{K}=\left[\begin{array}{ll}
k_{g g} & k_{g l} \\
k_{l g} & k_{l l}
\end{array}\right] ; \quad \mathbf{M}=\left[\begin{array}{cc}
m_{g g} & m_{g l} \\
m_{l g} & m_{l l}
\end{array}\right] ; \quad \mathbf{X}=\left\{\begin{array}{l}
x_{g} \\
x_{l}
\end{array}\right\} .
$$

Solution of the eigenvalue problem gives the frequency of significant cabinet mode and the corresponding eigenvector. Development of stiffness matrix $\mathbf{K}$ in case of a rocking mode requires some means of calculating the stiffness $k_{v}$ of equivalent vertical spring for a particular cabinet mounting arrangement. Simplified expressions for doing so in various types of cabinet mounting arrangement are presented in Yang et al. (2002). Such a simplified Ritz vector based method can be easily implemented either through hand calculations or even through a MATLAB based code. Initial development of Ritz vector approach has been validated by comparison with results presented above as evaluated from experimentally validated finite element analyses. However, this initial development is somewhat limited. Application to an actual plant would require additional work collecting relevant information, finite element analyses, validation with experimental data, and development of the code or spreadsheets for implementing the approach.

\section{COMPUTER PROGRAM INCABS}

INCABS implements the Ritz Vector approach described above. To use INCABS, only limited information on cabinet properties is required. The user needs to identify the number of side walls in cabinet which governs the presence of global cantilever modes. If a global rocking mode is present, an estimate of rotational stiffness at the cabinet base is needed. For cabinets with anchor bolt mounting configurations that are similar to one of the three mounting arrangements shown in Figs. 25 - 27, an estimate of the rotational stiffness can be calculated internally by the program. A user supplied value can also be input into the program for other types of bolt configurations. The user is required to identify the number of Ritz vectors that should be included in the analysis. The boundary conditions for the local modes are required to be identified. Guidance on selection of boundary conditions and the corresponding Ritz vectors is given below.

\section{$\underline{\text { Ritz Vectors }}$}

Accuracy of dynamic properties depends on the selection of Ritz vectors. The selection of Ritz vectors is governed by the boundary conditions. Several mathematical functions have been proposed as Ritz vectors by various researchers. INCABS uses simple and easy-to-use mathematical functions given in vibration/structural dynamics handbooks. For members having complex boundary conditions, more than one Ritz vector may be required. In such cases, the local mode can be expressed as a superposition of more than one Ritz vector. In addition, including higher-order Ritz vectors improves the accuracy of the calculated fundamental local mode. However, the computational complexity increases as increasing number of Ritz vectors are included in the analysis. In certain cases, such as frames, different members may 
require different types of Ritz vectors. Such a situation occurs primarily when the various members are not connected to each other except at their ends. Table 2 gives typical mathematical functions that can be used as Ritz vectors for a few idealized boundary conditions.

Table 2: Ritz Vectors for Fundamental Mode Shapes

\begin{tabular}{|c|c|}
\hline Description & Ritz Vector \\
\hline $\begin{array}{l}\text { Clamped-Clamped } \\
\phi_{\mathrm{cc}}\end{array}$ & $\cosh \frac{\lambda x}{L}-\cos \frac{\lambda x}{L}-\sigma\left(\sinh \frac{\lambda x}{L}-\sin \frac{\lambda x}{L}\right)$ \\
\hline $\begin{array}{c}\text { Pined-Pined } \\
\quad \phi_{\mathrm{pp}}\end{array}$ & $\sin \frac{\pi x}{L}$ \\
\hline $\begin{array}{l}\text { Clamped-Pined } \\
\phi_{\mathrm{cp}}\end{array}$ & $\begin{array}{c}\cosh \frac{\lambda x}{L}-\cos \frac{\lambda x}{L}-\sigma\left(\sinh \frac{\lambda x}{L}-\sin \frac{\lambda x}{L}\right) \\
\lambda=3.92660231, \sigma=1.000777304\end{array}$ \\
\hline $\begin{array}{l}\text { Clamped-Free } \\
\phi_{c f}\end{array}$ & $\begin{array}{c}\cosh \frac{\lambda x}{L}-\cos \frac{\lambda x}{L}-\sigma\left(\sinh \frac{\lambda x}{L}-\sin \frac{\lambda x}{L}\right) \\
\lambda=1.87510407, \sigma=0.734095514\end{array}$ \\
\hline $\begin{array}{l}\text { Sliding-Pined } \\
\phi_{\mathrm{sp}}\end{array}$ & $\cos \frac{\pi x}{2 L}$ \\
\hline $\begin{array}{l}\text { Clamped-Sliding } \\
\phi_{\mathrm{cs}}\end{array}$ & $\begin{array}{c}\cosh \frac{\lambda x}{L}-\cos \frac{\lambda x}{L}-\sigma\left(\sinh \frac{\lambda x}{L}=\sin \frac{\lambda x}{L}\right) \\
\lambda=2.36502037, \sigma=0.982502207\end{array}$ \\
\hline $\begin{array}{c}\text { Free-free } \\
\phi_{\mathrm{ff}}\end{array}$ & 1 \\
\hline
\end{tabular}

\section{Boundary Conditions}

For a plate, the boundary conditions at the four edges are governed by the torsional rigidity provided by the structural members at each of the four edges. When the plate is supported at its edge by a thin side wall, its out-of-plane movement is restrained by high in-plane stiffness of the side wall. The thin side wall does not offer a significant rotational restraint and the plate can undergo rotation at this edge. Therefore, the boundary condition at such an edge can be considered to be simply supported.

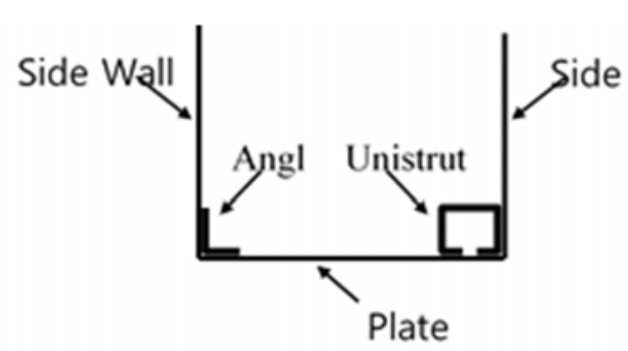

Figure 3. Plate Boundary Conditions

However, if a beam member (such as an angle or unistrut) is present at the edge of plate and supporting side wall, a rotational restraint may be present due to the torsional rigidity of the beam member. 
If the beam member has small torsional rigidity, the boundary condition for such an edge can be considered to be simply supported. If the structural member has high torsional rigidity, the boundary condition can be considered to be clamped. However, in most situations, the beam member provides partial rotational restraint to the plate. INCABS can account for the actual torsional stiffness of the beam member. The plate will be treated as a stiffened plate. The user should input the torsional constant $\mathrm{J}$ and select both the Ritz vectors corresponding to the simply supported and the clamped boundary conditions.

In some cases, a plate may be supported at its edge by only a beam (angle or unistrut) and not by a side wall. The beam member offers rotational restraint at the plate edge and allows the plate to undergo an outof-plane deflection as the beam is supported only at its ends. Therefore, if the structural member has high torsional rigidity, the plate edge can undergo an out-of-plane translation but no rotation. The boundary condition at such an edge can be considered to be a clamped edge on a roller. If the beam member has small torsional rigidity, the plate edge can undergo both translation and free rotation, resulting in a free edge boundary condition. Again, INCABS can account for the actual bending and torsional stiffness of the beam member. The user should input the moment of inertia $\mathrm{I}$, the torsional constant $\mathrm{J}$ of the beam member, and select both the Ritz vectors corresponding to the clamped edge on a roller and the free edge boundary conditions.

For a frame, the members are either bolted or welded at their ends. Welded connections, typically, offer high rotational restraint compared to the bolted connections. For the internal frame in cabinet DGLSB, the horizontal unistruts were welded at their two ends. Therefore, a clamped boundary condition was assumed at each end and produced accurate results. For bolted connections, however, a simply supported condition is recommended at each end.

\section{$\underline{\text { Rotational Stiffness, } \mathrm{K}_{\Theta}}$}

The value of rotational stiffness $\mathrm{K}_{\Theta}$ is obtained using equations for three different anchor bolt configurations. One of the two mounting arrangements has 12 anchor bolts and is shown in Figure 4. Rotational stiffness, $\mathrm{K}_{\ominus}$, for this mounting arrangement is obtained using the following equation.

$$
\begin{gathered}
\mathrm{K}_{\theta}=6.54\left(\sum_{i=1}^{N} c_{i}^{2}\right) D^{2} \frac{E t^{3}}{12\left(1-v^{2}\right)} \frac{1}{\Delta_{\mathrm{b}}^{2}} \\
0.1 \text { in } \leq t \leq 0.2 \text { in } \\
1.0 \text { in } \leq \Delta_{b} \leq 2.0 \text { in } \\
16 \text { in } \leq D \leq 24 \text { in }
\end{gathered}
$$




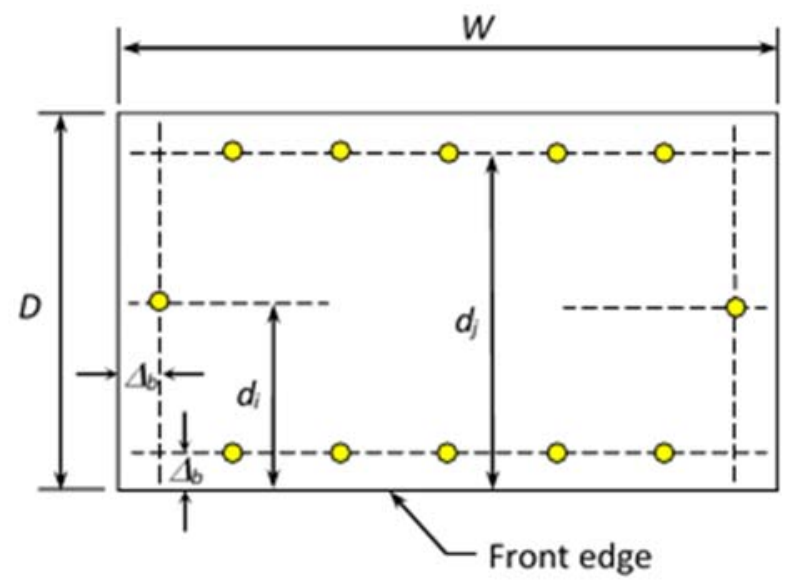

Figure 4. Blot Configuration 1

where $\mathrm{d}_{\mathrm{i}}$ is the distance of each bolt $\mathrm{i}$ from the front edge of base plate; $\Delta_{\mathrm{b}}$ is the distance of each bolt from the nearest base plate edge; $t$ is the base plate thickness; and $N$ the total number of bolts. $E$ and $v$ are Young's modulus and Poisson's ratio for base plate material, respectively.

The second mounting arrangement for which $\mathrm{K}_{\ominus}$ can be evaluated internally in INCABS, has 4 anchor bolts (Figure 5). $\mathrm{K}_{\ominus}$ for this mounting arrangement is obtained using the following equation.

$$
\mathrm{K}_{\theta}=9.27\left(\sum_{i=1}^{N} c_{i}^{2}\right) D^{2} \frac{E t^{3}}{12\left(1-v^{2}\right)} \frac{1}{\Delta_{\mathrm{b}}^{2}}
$$

0.1 in $\leq t \leq 0.2$ in

1.0 in $\leq \Delta_{\mathrm{b}} \leq 2.0$ in

16 in $\leq D \leq 24$ in

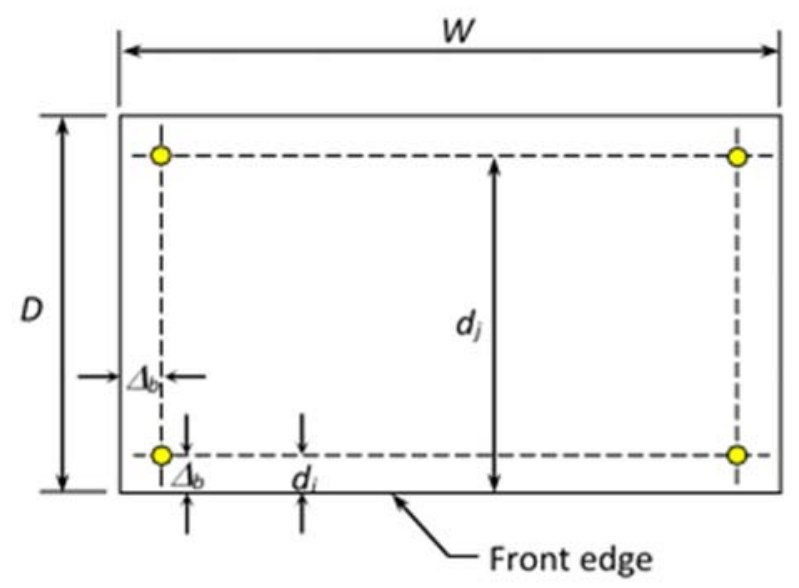

Figure 5. Blot Configuration 2 
Configuration 3 may consist of $\mathrm{m}$ bays in which triangular base plates are used for anchoring. The outer frame consists of channel sections and the bays are separated by tubular beams (Figure 6). $\mathrm{K}_{\theta}$ for this mounting arrangement is obtained using the following equation.

$$
\begin{gathered}
\mathrm{K}_{\theta}=2 K_{v} \sum_{i=1}^{2} d_{i}^{2}+(m-1)\left(2 K_{v} D^{2}+\frac{3 E I D^{2}}{L^{3}}\right) \\
\mathrm{K}_{\mathrm{v}}=16.44 \frac{E t^{3}}{12\left(1-v^{2}\right)} \frac{1}{\Delta_{b}^{2}} \\
0.2 \text { in } \leq t \leq 0.4 \text { in } \\
2.25 \text { in } \leq \Delta_{\mathrm{b}} \leq 3.25 \text { in } \\
25 \text { in } \leq D \leq 35 \text { in }
\end{gathered}
$$

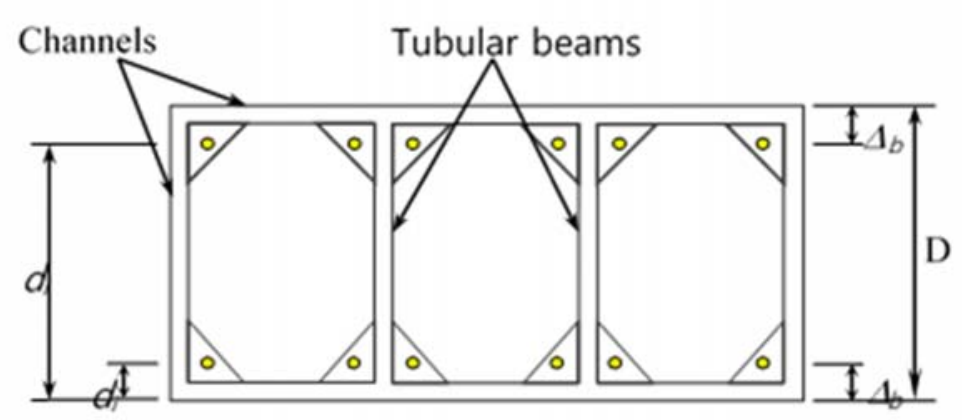

Figure 6. Bolt Configuration 3

\section{DISCUSSION}

It is recommended that the methodology in EPRI NP-7146 SL based on a constant amplification factors is not appropriate under the current practice for seismic qualification. The constant amplification factors can be excessively conservative in some cases thereby rendering many electrical systems or relays to be seismically unfit for use in a plant. In other cases, the constant amplification factors can be unconservative because of the use of clipping factors. Therefore, they can result in incorrect ICRS for such cases.

The Ritz-vector method is systematically implemented into INCABS for a user-friendly implementation. The Ritz-vector method can be a good alternative to the methodology in EPRI NP-7146 SL. However, application of this method to an actual plant would require additional work collecting relevant information, finite element analyses, validation with experimental data, and development of the code or spreadsheets for implementing the approach. 


\section{REFERENCES}

Electric Power Research Institute (EPRI) (1995), Guidelines for Development of In-Cabinet Seismic Demand for Devices Mounted in Electrical Cabinets, Technical report EPRI NP-7146 SL R1, CA, USA.

Gupta, A., Rustogi, S. K., Gupta, A. K. (1999). "Ritz vector approach for evaluating incabinet response spectra", Nuclear Engineering and Design, UK, 190 255-272.

Gupta, A., Yang, J. (2002). "Modified Ritz vector approach for dynamic properties of electrical cabinets and control panels", Nuclear Engineering and Design, UK, 217 49-62.

Kim, I. H., Park, G. S. (2016). "Field investigation of the damages due to the Sep 12 Gyeongju earthquake," Earthquake Engineering Society of Korea Special Session, Korea.

Korean Ministry of Public Safety and Security (KMPSS) (2016). "Damage recovery situation of earthquake on 9.12 and future plan," Seoul, Korea.

Korean Ministry of Land, Infrastructure and Transport (KMLIT) (2017). Korean Design Standard, Seoul, Korea

Sun, C. G., Oh, T. S., Park, J. H., Ji, H. C. (2016). "Seismic properties of the Sep 12 Gyeongju earthquake," Earthquake Engineering Society of Korea Special Session, Korea.

United States Nuclear Regulatory Commission (USNRC) (2012). Recommendation 2.1: Seismic, USA.

United States Nuclear Regulatory Commission (USNRC) (2014). Design response spectra for seismic design of nuclear power plants, Regulatory Guide, USA.

Yang, J., Rustogi, S. K., Gupta, A. (2002). "Rocking stiffness of mounting arrangements in electrical cabinets and control panels", Nuclear Engineering and Design, UK, 219 127-141. 